\title{
Commutator of fractional integral with Lipschitz functions associated with Schrödinger operator on local generalized Morrey spaces
}

\author{
Vagif S. Guliyev ${ }^{1,2,3}$ and Ali Akbulut ${ }^{* *}$ (1)
}

${ }^{*}$ Correspondence: akbulut72@gmail.com

${ }^{1}$ Department of Mathematics, Ahi Evran University, Kirsehir, Turkey Full list of author information is available at the end of the article

\begin{abstract}
Let $L=-\Delta+V$ be a Schrödinger operator on $\mathbb{R}^{n}$, where $n \geq 3$ and the nonnegative potential $V$ belongs to the reverse Hölder class $R H_{q_{1}}$ for some $q_{1}>n / 2$. Let $b$ belong to a new Campanato space $\Lambda_{\nu}^{\theta}(\rho)$ and $\mathcal{I}_{\beta}^{L}$ be the fractional integral operator associated with $L$. In this paper, we study the boundedness of the commutators $\left[b, \mathcal{I}_{\beta}^{L}\right]$ with $b \in \Lambda_{\nu}^{\theta}(\rho)$ on local generalized Morrey spaces $L M_{p, \varphi}^{\alpha, V,\left\{x_{0}\right\}}$, generalized Morrey spaces $M_{p, \varphi}^{\alpha, V}$ and vanishing generalized Morrey spaces $V M_{p, \varphi}^{\alpha, V}$ associated with Schrödinger operator, respectively. When $b$ belongs to $\Lambda_{v}^{\theta}(\rho)$ with $\theta>0,0<v<1$ and $\left(\varphi_{1}, \varphi_{2}\right)$ satisfies some conditions, we show that the commutator operator $\left[b, \mathcal{I}_{\beta}^{L}\right]$ are bounded from $L M_{p, \varphi_{1}}^{\alpha, V,\left\{x_{0}\right\}}$ to $L M_{q, \varphi_{2}}^{\alpha, V,\left\{x_{0}\right\}}$, from $M_{p, \varphi_{1}}^{\alpha, V}$ to $M_{q, \varphi_{2}}^{\alpha, V}$ and from $V M_{p, \varphi_{1}}^{\alpha, V}$ to $V M_{q, \varphi_{2}}^{\alpha, V}, 1 / p-1 / q=(\beta+v) / n$.

MSC: $42 \mathrm{~B} 35 ; 35 \mathrm{~J} 10 ; 47 \mathrm{H} 50$
\end{abstract}

Keywords: Schrödinger operator; Fractional integral; Commutator; Lipschitz function; Local generalized Morrey space

\section{Introduction and main results}

Let us consider the Schrödinger operator

$$
L=-\Delta+V \quad \text { on } \mathbb{R}^{n}, n \geq 3
$$

where $V$ is a nonnegative, $V \neq 0$, and belongs to the reverse Hölder class $R H_{q}$ for some $q \geq n / 2$, i.e., there exists a constant $C>0$ such that the reverse Hölder inequality

$$
\left(\frac{1}{|B(x, r)|} \int_{B(x, r)} V^{q}(y) d y\right)^{1 / q} \leq \frac{C}{|B(x, r)|} \int_{B(x, r)} V(y) d y
$$

holds for every $x \in \mathbb{R}^{n}$ and $0<r<\infty$, where $B(x, r)$ denotes the ball centered at $x$ with radius $r$. In particular, if $V$ is a nonnegative polynomial, then $V \in R H_{\infty}$.

(c) The Author(s) 2018. This article is distributed under the terms of the Creative Commons Attribution 4.0 International License (http://creativecommons.org/licenses/by/4.0/), which permits unrestricted use, distribution, and reproduction in any medium, provided you give appropriate credit to the original author(s) and the source, provide a link to the Creative Commons license, and indicate if changes were made. 
As in [29], for a given potential $V \in R H_{q}$ with $q \geq n / 2$, we define the auxiliary function

$$
\rho(x):=\frac{1}{m_{V}(x)}=\sup _{r>0}\left\{r: \frac{1}{r^{n-2}} \int_{B(x, r)} V(y) d y \leq 1\right\} .
$$

It is well known that $0<\rho(x)<\infty$ for any $x \in \mathbb{R}^{n}$.

Let $\theta>0$ and $0<v<1$, in view of [22], the Campanato class, associated with the Schrödinger operator $\Lambda_{v}^{\theta}(\rho)$ consists of the locally integrable functions $b$ such that

$$
\frac{1}{|B(x, r)|^{1+v / n}} \int_{B(x, r)}\left|b(y)-b_{B}\right| d y \leq C\left(1+\frac{r}{\rho(x)}\right)^{\theta}
$$

for all $x \in \mathbb{R}^{n}$ and $r>0$. A seminorm of $b \in \Lambda_{v}^{\theta}(\rho)$, denoted by $[b]_{\beta}^{\theta}$, is given by the infimum of the constants in the inequality above.

Note that if $\theta=0, \Lambda_{v}^{\theta}(\rho)$ is the classical Campanato space; if $v=0, \Lambda_{v}^{\theta}(\rho)$ is exactly the space $B M O_{\theta}(\rho)$ introduced in [5].

We now present the definition of generalized Morrey spaces $M_{p, \varphi}^{\alpha, V}\left(\mathbb{R}^{n}\right)$ (including the weak version) associated with a Schrödinger operator, which was introduced by the first author in [18].

The classical Morrey spaces $L_{p, \lambda}\left(\mathbb{R}^{n}\right)$ was introduced by Morrey in [24] to study the local behavior of solutions to second order elliptic partial differential equations. For the properties and applications of classical Morrey spaces, we refer the reader to [9-12, 24, 35]. The generalized Morrey spaces are defined with $r^{\lambda}$ replaced by a general nonnegative function $\varphi(x, r)$ satisfying some assumptions (see, for example, $[15,23,25,30])$.

For brevity, in the sequel we use the notations

$$
\mathfrak{A}_{p, \varphi}^{\alpha, V}(f ; x, r):=\left(1+\frac{r}{\rho(x)}\right)^{\alpha} r^{-n / p} \varphi(x, r)^{-1}\|f\|_{L_{p}(B(x, r))}
$$

and

$$
\mathfrak{A}_{\Phi, \varphi}^{W, \alpha, V}(f ; x, r):=\left(1+\frac{r}{\rho(x)}\right)^{\alpha} r^{-n / p} \varphi(x, r)^{-1}\|f\|_{W L_{p}(B(x, r))} .
$$

Definition 1.1 Let $\varphi(x, r)$ be a positive measurable function on $\mathbb{R}^{n} \times(0, \infty), 1 \leq p<\infty$, $\alpha \geq 0$, and $V \in R H_{q}, q \geq 1$. For any fixed $x_{0} \in \mathbb{R}^{n}$ we denote by $L M_{p, \varphi}^{\alpha, V,\left\{x_{0}\right\}}=L M_{p, \varphi}^{\alpha, V,\left\{x_{0}\right\}}\left(\mathbb{R}^{n}\right)$ the local generalized Morrey space associated with Schrödinger operator, the space of all functions $f \in L_{p}^{l o c}\left(\mathbb{R}^{n}\right)$ with finite norm

$$
\|f\|_{L M_{p, \varphi}^{\alpha, V,\left\{x_{0}\right\}}}=\sup _{r>0} \mathfrak{A}_{p, \varphi}^{\alpha, V}\left(f ; x_{0}, r\right) .
$$

Also $W L M_{p, \varphi}^{\alpha, V,\left\{x_{0}\right\}}=W L M_{p, \varphi}^{\alpha, V,\left\{x_{0}\right\}}\left(\mathbb{R}^{n}\right)$ we denote the weak local generalized Morrey space associated with Schrödinger operator, the space of all functions $f \in W L_{p}^{l o c}\left(\mathbb{R}^{n}\right)$ with

$$
\|f\|_{W L M_{p, \varphi}^{\alpha, V,\left\{x_{0}\right\}}}=\sup _{r>0} \mathfrak{A}_{p, \varphi}^{W, \alpha, V}\left(f ; x_{0}, r\right)<\infty
$$


The local spaces $L M_{p, \varphi}^{\alpha, V,\left\{x_{0}\right\}}\left(\mathbb{R}^{n}\right)$ and $W L M_{p, \varphi}^{\alpha, V,\left\{x_{0}\right\}}\left(\mathbb{R}^{n}\right)$ are Banach spaces with respect to the norm

$$
\|f\|_{L M_{p, \varphi}^{\alpha, V,\left\{x_{0}\right\}}}=\sup _{r>0} \mathfrak{A}_{p, \varphi}^{\alpha, V}\left(f ; x_{0}, r\right), \quad\|f\|_{W L M_{p, \varphi}^{\alpha, V,\left\{x_{0}\right\}}}=\sup _{r>0} \mathfrak{A}_{p, \varphi}^{W, \alpha, V}\left(f ; x_{0}, r\right),
$$

respectively.

\section{Remark 1.1}

(i) When $\alpha=0$, and $\varphi(x, r)=r^{(\lambda-n) / p}, L M_{p, \varphi}^{\alpha, V,\left\{x_{0}\right\}}\left(\mathbb{R}^{n}\right)$ is the local (central) Morrey space $L M_{p, \lambda}^{\{0\}}\left(\mathbb{R}^{n}\right)$ studied in [4].

(ii) When $\alpha=0, L M_{p, \varphi}^{\alpha, V,\left\{x_{0}\right\}}\left(\mathbb{R}^{n}\right)$ is the local generalized Morrey space $V M_{p, \varphi}^{\left\{x_{0}\right\}}\left(\mathbb{R}^{n}\right)$ were introduced by the first author in [13]; see also [14, 16, 21] etc.

Definition 1.2 The vanishing generalized Morrey space associated with the Schrödinger operator $V M_{p, \varphi}^{\alpha, V}\left(\mathbb{R}^{n}\right)$ is defined as the spaces of functions $f \in M_{p, \varphi}^{\alpha, V}\left(\mathbb{R}^{n}\right)$ such that

$$
\lim _{r \rightarrow 0} \sup _{x \in \mathbb{R}^{n}} \mathfrak{A}_{p, \varphi}^{\alpha, V}(f ; x, r)=0
$$

The vanishing weak generalized Morrey space associated with the Schrödinger operator $V W M_{p, \varphi}^{\alpha, V}\left(\mathbb{R}^{n}\right)$ is defined as the spaces of functions $f \in W M_{p, \varphi}^{\alpha, V}\left(\mathbb{R}^{n}\right)$ such that

$$
\lim _{r \rightarrow 0} \sup _{x \in \mathbb{R}^{n}} \mathfrak{A}_{p, \varphi}^{W, \alpha, V}(f ; x, r)=0
$$

The vanishing spaces $V M_{p, \varphi}^{\alpha, V}\left(\mathbb{R}^{n}\right)$ and $V W M_{p, \varphi}^{\alpha, V}\left(\mathbb{R}^{n}\right)$ are Banach spaces with respect to the norm

$$
\begin{aligned}
& \|f\|_{V M_{p, \varphi}^{\alpha, V}} \equiv\|f\|_{M_{p, \varphi}^{\alpha}}^{\alpha, V}=\sup _{x \in \mathbb{R}^{n}, r>0} \mathfrak{A}_{p, \varphi}^{\alpha, V}(f ; x, r), \\
& \|f\|_{V W M_{p, \varphi}^{\alpha, V}} \equiv\|f\|_{W M_{p, \varphi}^{\alpha, V}}=\sup _{x \in \mathbb{R}^{n}, r>0} \mathfrak{A}_{W, p, \varphi}^{\alpha, V}(f ; x, r),
\end{aligned}
$$

respectively.

In the case $\alpha=0$, and $\varphi(x, r)=r^{(\lambda-n) / p} V M_{p, \varphi}^{\alpha, V}\left(\mathbb{R}^{n}\right)$ is the vanishing Morrey space $V M_{p, \lambda}$ introduced in [33], where applications to PDE were considered.

We refer to $[3,20,27,28]$ for some properties of vanishing generalized Morrey spaces.

Definition 1.3 Let $L=-\Delta+V$ with $V \in R H_{q_{1}}, q_{1}>n / 2$. The fractional integral associated with $L$ is defined by

$$
\mathcal{I}_{\beta}^{L} f(x)=L^{-\beta / 2} f(x)=\int_{0}^{\infty} e^{-t L}(f)(x) t^{\beta / 2-1} d t
$$

for $0<\beta<n$. The commutator of $\mathcal{I}_{\beta}^{L}$ is defined by

$$
\left[b, \mathcal{I}_{\beta}^{L}\right] f(x)=b(x) \mathcal{I}_{\beta}^{L} f(x)-\mathcal{I}_{\beta}^{L}(b f)(x) .
$$


Note that, if $L=-\Delta$ is the Laplacian on $\mathbb{R}^{n}$, then $\mathcal{I}_{\beta}^{L}$ and $\left[b, \mathcal{I}_{\beta}^{L}\right]$ are the Riesz potential $I_{\beta}$ and the commutator of the Riesz potential $\left[b, I_{\beta}\right]$, respectively, that is,

$$
I_{\beta} f(x)=\int_{\mathbb{R}^{n}} \frac{f(y)}{|x-y|^{n}} d y, \quad\left[b, I_{\beta}\right] f(x)=\int_{\mathbb{R}^{n}} \frac{b(x)-b(y)}{|x-y|^{n}} f(y) d y .
$$

When $b \in B M O$, Chanillo proved in [8] that $\left[b, I_{\beta}\right]$ is bounded from $L_{p}\left(\mathbb{R}^{n}\right)$ to $L_{q}\left(\mathbb{R}^{n}\right)$ with $1 / q=1 / p-\beta / n, 1<p<n / \beta$. When $b$ belongs to the Campanato space $\Lambda_{v}, 0<v<1$, Paluszynski in [26] showed that $\left[b, I_{\beta}\right]$ is bounded from $L_{p}\left(\mathbb{R}^{n}\right)$ to $L_{q}\left(\mathbb{R}^{n}\right)$ with $1 / q=1 / p-$ $(\beta+v) / n, 1<p<n /(\beta+v)$. When $b \in B M O_{\theta}(\rho)$, Bui in [6] obtained the boundedness of $\left[b, \mathcal{I}_{\beta}^{L}\right]$ from $L_{p}\left(\mathbb{R}^{n}\right)$ to $L_{q}\left(\mathbb{R}^{n}\right)$ with $1 / q=1 / p-\beta / n, 1<p<n / \beta$.

Inspired by the above results, we are interested in the boundedness of $\left[b, \mathcal{I}_{\beta}^{L}\right]$ on generalized Morrey spaces $M_{p, \varphi}^{\alpha, V}\left(\mathbb{R}^{n}\right)$ and the vanishing generalized Morrey spaces $V M_{p, \varphi}^{\alpha, V}\left(\mathbb{R}^{n}\right)$, when $b$ belongs to the new Campanato class $\Lambda_{v}^{\theta}(\rho)$.

In this paper, we consider the boundedness of the commutator of $\mathcal{I}_{\beta}^{L}$ on the local generalized Morrey spaces $L M_{p, \varphi}^{\alpha, V,\left\{x_{0}\right\}}$, the generalized Morrey spaces $M_{p, \varphi}^{\alpha, V}\left(\mathbb{R}^{n}\right)$ and the vanishing generalized Morrey spaces $V M_{p, \varphi}^{\alpha, V}\left(\mathbb{R}^{n}\right)$. When $b$ belongs to the new Campanato space $\Lambda_{v}^{\theta}(\rho), 0<v<1$, we show that $\left[b, \mathcal{I}_{\beta}^{L}\right]$ are bounded from $L M_{p, \varphi 1}^{\alpha, V,\left\{x_{0}\right\}}$ to $L M_{q, \varphi_{2}}^{\alpha, V,\left\{x_{0}\right\}}$, from $M_{p, \varphi}^{\alpha, V}\left(\mathbb{R}^{n}\right)$ to $M_{q, \varphi}^{\alpha, V}\left(\mathbb{R}^{n}\right)$ and from $V M_{p, \varphi}^{\alpha, V}\left(\mathbb{R}^{n}\right)$ to $V M_{q, \varphi}^{\alpha, V}\left(\mathbb{R}^{n}\right)$ with $1 / q=1 / p-(\beta+v) / n$, $1<p<n /(\beta+v)$.

Our main results are as follows.

Theorem 1.1 Let $x_{0} \in \mathbb{R}^{n}, b \in \Lambda_{v}^{\theta}(\rho), V \in R H_{q_{1}}, q_{1}>n / 2,0<v<1, \alpha \geq 0,1 \leq p<n /(\beta+$ v), $1 / q=1 / p-(\beta+v) / n$ and let $\varphi_{1}, \varphi_{2} \in \Omega_{p, l o c}^{\alpha, V}$ satisfy the condition

$$
\int_{r}^{\infty} \frac{\operatorname{essinf}_{t<s<\infty} \varphi_{1}\left(x_{0}, s\right) s^{\frac{n}{p}}}{t^{\frac{n}{q}}} \frac{d t}{t} \leq c_{0} \varphi_{2}\left(x_{0}, r\right)
$$

where $c_{0}$ does not depend on $r$. Then the operator $\left[b, \mathcal{I}_{\beta}^{L}\right]$ is bounded from $L M_{p, \varphi_{1}}^{\alpha, V,\left\{x_{0}\right\}}$ to $L M_{q, \varphi_{2}}^{\alpha, V,\left\{x_{0}\right\}}$ for $p>1$ and from $L M_{1, \varphi_{1}}^{\alpha, V,\left\{x_{0}\right\}}$ to $W L M_{\frac{n}{n-\beta-\nu}, \varphi_{2}}^{\alpha, V,\left\{x_{0}\right\}}$. Moreover, for $p>1$

$$
\left\|\left[b, \mathcal{I}_{\beta}^{L}\right] f\right\|_{L M_{q, \varphi_{2}}^{\alpha, V,\left\{x_{0}\right\}}} \leq C[b]_{v}^{\theta}\|f\|_{L M_{p, \varphi_{1}}^{\alpha, V,\left\{x_{0}\right\}}}
$$

and for $p=1$

$$
\left\|\left[b, \mathcal{I}_{\beta}^{L}\right] f\right\|_{W L M^{\frac{n}{n}, V,\left\{x_{0}\right\}}, \varphi_{2}}^{n-\beta-v} \leq C[b]_{v}^{\theta}\|f\|_{L M_{1, \varphi_{1}}^{\alpha,\left\{, x_{0}\right\}}}
$$

where $C$ does not depend on $f$.

Corollary 1.1 Let $b \in \Lambda_{v}^{\theta}(\rho), V \in R H_{q_{1}}, q_{1}>n / 2,0<v<1, \alpha \geq 0,1 \leq p<n /(\beta+v), 1 / q=$ $1 / p-(\beta+v) / n$ and let $\varphi_{1} \in \Omega_{p}^{\alpha, V}, \varphi_{2} \in \Omega_{q}^{\alpha, V}$ satisfy the condition

$$
\int_{r}^{\infty} \frac{\operatorname{essinf}_{t<s<\infty} \varphi_{1}(x, s) s^{\frac{n}{p}}}{t^{\frac{n}{q}}} \frac{d t}{t} \leq c_{0} \varphi_{2}(x, r),
$$


where $c_{0}$ does not depend on $x$ and $r$. Then the operator $\left[b, \mathcal{I}_{\beta}^{L}\right]$ is bounded from $M_{p, \varphi_{1}}^{\alpha, V}$ to $M_{q, \varphi_{2}}^{\alpha, V}$ for $p>1$ and from $M_{1, \varphi_{1}}^{\alpha, V}$ to $W M_{\frac{n}{n-\beta-v}, \varphi_{2}}^{\alpha, V}$. Moreover, for $p>1$

$$
\left\|\left[b, \mathcal{I}_{\beta}^{L}\right] f\right\|_{M_{q_{,}, V}^{\alpha}}^{\alpha, V} \leq C[b]_{\theta}\|f\|_{M_{p, \varphi_{1}}^{\alpha, V}},
$$

and for $p=1$

$$
\left\|\left[b, \mathcal{I}_{\beta}^{L}\right] f\right\|_{W M_{\frac{n}{n, V_{n}, \varphi_{2}}}^{\alpha-\beta-\nu}} \leq C\|f\|_{M_{1, \varphi_{1}}^{\alpha, V}}
$$

where $C$ does not depend on $f$.

Theorem 1.2 Let $b \in \Lambda_{v}^{\theta}(\rho), V \in R H_{q_{1}}, q_{1}>n / 2,0<v<1, \alpha \geq 0, b \in \Lambda_{v}^{\theta}(\rho), 1<p<n /(\beta+$ v), $1 / q=1 / p-(\beta+v) / n$, and let $\varphi_{1} \in \Omega_{p, 1}^{\alpha, V}, \varphi_{2} \in \Omega_{q, 1}^{\alpha, V}$ satisfy the conditions

$$
c_{\delta}:=\int_{\delta}^{\infty} \sup _{x \in \mathbb{R}^{n}} \varphi_{1}(x, t) \frac{d t}{t}<\infty
$$

for every $\delta>0$, and

$$
\int_{r}^{\infty} \varphi_{1}(x, t) \frac{d t}{t^{1-\beta-\nu}} \leq C_{0} \varphi_{2}(x, r)
$$

where $C_{0}$ does not depend on $x \in \mathbb{R}^{n}$ and $r>0$. Then the operator $\left[b, \mathcal{I}_{\beta}^{L}\right]$ is bounded from $V M_{p, \varphi_{1}}^{\alpha, V}$ to $V M_{q, \varphi_{2}}^{\alpha, V}$ for $p>1$ and from $V M_{1, \varphi_{1}}^{\alpha, V}$ to $V W M_{\frac{n}{n-\beta-v}, \varphi_{2}}^{\alpha, V}$.

Remark 1.2 Note that, in the case of $V \equiv 0, v=0$ Corollary 1.1 and Theorem 1.2 were proved in [19, Corollary 5.5 and 7.5] and in the case of $\varphi(x, r)=r^{(\lambda-n) / p}, v=0$ in [32, Theorems 1.3 and 1.4].

In this paper, we shall use the symbol $A \lesssim B$ to indicate that there exists a universal positive constant $C$, independent of all important parameters, such that $A \leq C B . A \approx B$ means that $A \lesssim B$ and $B \lesssim A$.

\section{Some technical lemmas and propositions}

We would like to recall the important properties concerning the critical function.

Lemma 2.1 ([29]) Let $V \in R H_{q_{1}}$ with $q_{1}>n / 2$. For the associated function $\rho$ there exist $C$ and $k_{0} \geq 1$ such that

$$
C^{-1} \rho(x)\left(1+\frac{|x-y|}{\rho(x)}\right)^{-k_{0}} \leq \rho(y) \leq C \rho(x)\left(1+\frac{|x-y|}{\rho(x)}\right)^{\frac{k_{0}}{1+k_{0}}}
$$

for all $x, y \in \mathbb{R}^{n}$.

Lemma 2.2 ([2]) Suppose $x \in B\left(x_{0}, r\right)$. Then for $k \in N$ we have

$$
\frac{1}{\left(1+\frac{2^{k} r}{\rho(x)}\right)^{N}} \lesssim \frac{1}{\left(1+\frac{2^{k} r}{\rho\left(x_{0}\right)}\right)^{N /\left(k_{0}+1\right)}}
$$


According to [5], the new BMO space $B M O_{\theta}(\rho)$ with $\theta \geq 0$ is defined as a set of all locally integrable functions $b$ such that

$$
\frac{1}{|B(x, r)|} \int_{B(x, r)}\left|b(y)-b_{B}\right| d y \leq C\left(1+\frac{r}{\rho(x)}\right)^{\theta}
$$

for all $x \in \mathbb{R}^{n}$ and $r>0$, where $b_{B}=\frac{1}{|B|} \int_{B} b(y) d y$. A norm for $b \in B M O_{\theta}(\rho)$, denoted by $[b]_{\theta}$, is given by the infimum of the constants in the inequalities above. Clearly, $B M O \subset$ $B M O_{\theta}(\rho)$.

Let $\theta>0$ and $0<v<1$, a seminorm on the Campanato class $\Lambda_{v}^{\theta}(\rho)$ is denoted by $[b]_{v}^{\theta}$,

$$
[b]_{\nu}^{\theta}:=\sup _{x \in \mathbb{R}^{n}, r>0} \frac{\frac{1}{|B(x, r)|^{1+\nu / n}} \int_{B(x, r)}\left|b(y)-b_{B}\right| d y}{\left(1+\frac{r}{\rho(x)}\right)^{\theta}}<\infty .
$$

The Lipschitz space, associated with the Schrödinger operator (see [22]), consists of the functions $f$ satisfying

$$
\|f\|_{\operatorname{Lip}_{\nu}^{\theta}(\rho)}:=\sup _{x \in \mathbb{R}^{n}, r>0} \frac{|f(x)-f(y)|}{|x-y|^{\nu}\left(1+\frac{|x-y|}{\rho(x)}+\frac{|x-y|}{\rho(y)}\right)^{\theta}}<\infty .
$$

It is easy to see that this space is exactly the Lipschitz space when $\theta=0$.

Note that if $\theta=0$ in (1.2), $\Lambda_{v}^{\theta}(\rho)$ is exactly the classical Campanato space; if $v=0, \Lambda_{v}^{\theta}(\rho)$ is exactly the space $B M O_{\theta}(\rho)$; if $\theta=0$ and $v=0$, it is exactly the John-Nirenberg space $B M O$.

The following relations between $\operatorname{Lip}_{v}^{\theta}(\rho)$ and $\Lambda_{v}^{\theta}(\rho)$ were proved in [22, Theorem 5].

Lemma 2.3 ([22]) Let $\theta>0$ and $0<v<1$. Then following embedding is valid:

$$
\Lambda_{v}^{\theta}(\rho) \subseteq \operatorname{Lip}_{v}^{\theta}(\rho) \subseteq \Lambda_{v}^{\left(k_{0}+1\right) \theta}(\rho)
$$

where $k_{0}$ is the constant appearing in Lemma 2.1.

We give some inequalities about the Campanato space, associated with the Schrödinger operator $\Lambda_{v}^{\theta}(\rho)$.

Lemma 2.4 ([22]) Let $\theta>0$ and $1 \leq s<\infty$. If $b \in \Lambda_{v}^{\theta}(\rho)$, then there exists a positive constant $C$ such that

$$
\left(\frac{1}{|B|} \int_{B}\left|b(y)-b_{B}\right|^{s} d y\right)^{1 / s} \leq C[b]_{v}^{\theta} r^{v}\left(1+\frac{r}{\rho(x)}\right)^{\theta^{\prime}}
$$

for all $B=B(x, r)$, with $x \in \mathbb{R}^{n}$ and $r>0$, where $\theta^{\prime}=\left(k_{0}+1\right) \theta$ and $k_{0}$ is the constant appearing in (2.1).

Let $K_{\beta}$ be the kernel of $\mathcal{I}_{\beta}^{L}$. The following result gives the estimate on the kernel $K_{\beta}(x, y)$. 
Lemma 2.5 ([6]) If $V \in R H_{q_{1}}$ with $q_{1}>n / 2$, then, for every $N$, there exists a constant $C$ such that

$$
\left|K_{\beta}(x, y)\right| \leq \frac{C}{\left(1+\frac{|x-y|}{\rho(x)}\right)^{N}} \frac{1}{|x-y|^{n-\beta}} .
$$

Finally, we recall a relationship between an essential supremum and an essential infimum.

Lemma 2.6 ([34]) Letf be a real-valued nonnegative function and measurable on $E$. Then

$$
\left(\underset{x \in E}{\operatorname{essinf} f(x))^{-1}}=\underset{x \in E}{\operatorname{ess} \sup } \frac{1}{f(x)}\right.
$$

It is natural, first of all, to find conditions ensuring that the spaces $L M_{p, \varphi}^{\alpha, V,\left\{x_{0}\right\}}$ and $M_{p, \varphi}^{\alpha, V}$ are nontrivial, that is, consist not only of functions equivalent to 0 on $\mathbb{R}^{n}$.

Lemma 2.7 Let $x_{0} \in \mathbb{R}^{n}, \varphi(x, r)$ be a positive measurable function on $\mathbb{R}^{n} \times(0, \infty), 1 \leq p<$ $\infty, \alpha \geq 0$, and $V \in R H_{q}, q \geq 1$. If

$$
\sup _{t<r<\infty}\left(1+\frac{r}{\rho\left(x_{0}\right)}\right)^{\alpha} \frac{r^{-\frac{n}{p}}}{\varphi\left(x_{0}, r\right)}=\infty \text { for some } t>0 \text {, }
$$

then $L M_{p, \varphi}^{\alpha, V,\left\{x_{0}\right\}}\left(\mathbb{R}^{n}\right)=\Theta$, where $\Theta$ is the set of all functions equivalent to 0 on $\mathbb{R}^{n}$.

Proof Let (2.4) be satisfied and $f$ be not equivalent to zero. Then $\|f\|_{L_{p}\left(B\left(x_{0}, t\right)\right)}>0$, hence

$$
\begin{aligned}
\|f\|_{L M_{p, \varphi}^{\alpha, V,\left\{x_{0}\right\}}} & \geq \sup _{t<r<\infty}\left(1+\frac{r}{\rho\left(x_{0}\right)}\right)^{\alpha} \varphi\left(x_{0}, r\right)^{-1} r^{-\frac{n}{p}}\|f\|_{L_{p}\left(B\left(x_{0}, r\right)\right)} \\
& \geq\|f\|_{L_{p}\left(B\left(x_{0}, t\right)\right)} \sup _{t<r<\infty}\left(1+\frac{r}{\rho\left(x_{0}\right)}\right)^{\alpha} \varphi\left(x_{0}, r\right)^{-1} r^{-\frac{n}{p}} .
\end{aligned}
$$

Therefore $\|f\|_{L M_{p, \varphi}^{\alpha, V,\left\{x_{0}\right\}}}=\infty$.

Remark 2.1 We denote by $\Omega_{p, l o c}^{\alpha, V}$ the sets of all positive measurable functions $\varphi$ on $\mathbb{R}^{n} \times$ $(0, \infty)$ such that, for all $t>0$,

$$
\sup _{x \in \mathbb{R}^{n}}\left\|\left(1+\frac{r}{\rho(x)}\right)^{\alpha} \frac{r^{-\frac{n}{p}}}{\varphi(x, r)}\right\|_{L_{\infty}(t, \infty)}<\infty .
$$

In what follows, keeping in mind Lemma 2.7, for the non-triviality of the space $L M_{p, \varphi}^{\alpha, V,\left\{x_{0}\right\}}\left(\mathbb{R}^{n}\right)$ we always assume that $\varphi \in \Omega_{p, l o c}^{\alpha, V}$.

Lemma 2.8 ([2]) Let $\varphi(x, r)$ be a positive measurable function on $\mathbb{R}^{n} \times(0, \infty), 1 \leq p<\infty$, $\alpha \geq 0$, and $V \in R H_{q}, q \geq 1$.

(i) If

$$
\sup _{t<r<\infty}\left(1+\frac{r}{\rho(x)}\right)^{\alpha} \frac{r^{-\frac{n}{p}}}{\varphi(x, r)}=\infty \text { for some } t>0 \text { and for all } x \in \mathbb{R}^{n}
$$


then $M_{p, \varphi}^{\alpha, V}\left(\mathbb{R}^{n}\right)=\Theta$.

(ii) If

$$
\sup _{0<r<\tau}\left(1+\frac{r}{\rho(x)}\right)^{\alpha} \varphi(x, r)^{-1}=\infty \quad \text { for some } \tau>0 \text { and for all } x \in \mathbb{R}^{n}
$$

then $M_{p, \varphi}^{\alpha, V}\left(\mathbb{R}^{n}\right)=\Theta$.

Remark 2.2 We denote by $\Omega_{p}^{\alpha, V}$ the sets of all positive measurable functions $\varphi$ on $\mathbb{R}^{n} \times$ $(0, \infty)$ such that, for all $t>0$,

$$
\begin{aligned}
& \sup _{x \in \mathbb{R}^{n}}\left\|\left(1+\frac{r}{\rho(x)}\right)^{\alpha} \frac{r^{-\frac{n}{p}}}{\varphi(x, r)}\right\|_{L_{\infty}(t, \infty)}<\infty, \quad \text { and } \\
& \sup _{x \in \mathbb{R}^{n}}\left\|\left(1+\frac{r}{\rho(x)}\right)^{\alpha} \varphi(x, r)^{-1}\right\|_{L_{\infty}(0, t)}<\infty,
\end{aligned}
$$

respectively. In what follows, keeping in mind Lemma 2.8, for the non-triviality of the space $M_{p, \varphi}^{\alpha, V}\left(\mathbb{R}^{n}\right)$ we always assume that $\varphi \in \Omega_{p}^{\alpha, V}$.

Remark 2.3 We denote by $\Omega_{p, 1}^{\alpha, V}$ the sets of all positive measurable functions $\varphi$ on $\mathbb{R}^{n} \times$ $(0, \infty)$ such that

$$
\inf _{x \in \mathbb{R}^{n}} \inf _{r>\delta}\left(1+\frac{r}{\rho(x)}\right)^{-\alpha} \varphi(x, r)>0, \quad \text { for some } \delta>0,
$$

and

$$
\lim _{r \rightarrow 0}\left(1+\frac{r}{\rho(x)}\right)^{\alpha} \frac{r^{n / p}}{\varphi(x, r)}=0
$$

For the non-triviality of the space $V M_{p, \varphi}^{\alpha, V}\left(\mathbb{R}^{n}\right)$ we always assume that $\varphi \in \Omega_{p, 1}^{\alpha, V}$.

\section{Proof of Theorem 1.1}

We first prove the following conclusions.

Lemma 3.1 Let $0<v<1,0<\beta+v<n$ and $b \in \Lambda_{v}^{\theta}(\rho)$, then the following pointwise estimate holds:

$$
\left|\left[b, \mathcal{I}_{\beta}^{L}\right] f(x)\right| \lesssim[b]_{v}^{\theta} I_{\beta+v}(|f|)(x)
$$

Proof Note that

$$
\begin{aligned}
{\left[b, \mathcal{I}_{\beta}^{L}\right] f(x) } & =b(x) \mathcal{I}_{\beta}^{L}(f)(x)-\mathcal{I}_{\beta}^{L}(b f)(x) \\
& =\int_{\mathbb{R}^{n}}[b(x)-b(y)] K_{\beta}(x, y) f(y) d y .
\end{aligned}
$$


If $b \in \Lambda_{v}^{\theta}(\rho)$, then from Lemma 2.5 we have

$$
\begin{aligned}
\left|\left[b, \mathcal{I}_{\beta}^{L}\right] f(x)\right| & \leq \int_{\mathbb{R}^{n}}|b(x)-b(y)|\left|K_{\beta}(x, y)\right||f(y)| d y \\
& \lesssim[b]_{v}^{\theta} \int_{\mathbb{R}^{n}}|x-y|^{v}\left|K_{\beta}(x, y)\right||f(y)| d y \\
& =[b]_{v}^{\theta} I_{\beta+\nu}(|f|)(x) .
\end{aligned}
$$

From Lemma 3.1 we get the following.

Corollary 3.1 Suppose $V \in R H_{q_{1}}$ with $q_{1}>n / 2$ and $b \in \Lambda_{v}^{\theta}(\rho)$ with $0<v<1$. Let $0<\beta+v<$ $n$ and let $1 \leq p<q<\infty$ satisfy $1 / q=1 / p-(\beta+v) / n$. Then for all $f$ in $L_{p}\left(\mathbb{R}^{n}\right)$ we have

$$
\left\|\left[b, \mathcal{I}_{\beta}^{L}\right] f\right\|_{L_{q}\left(\mathbb{R}^{n}\right)} \lesssim\|f\|_{L_{p}\left(\mathbb{R}^{n}\right)}
$$

when $p>1$, and also

$$
\left\|\left[b, \mathcal{I}_{\beta}^{L}\right] f\right\|_{W L_{q}\left(\mathbb{R}^{n}\right)} \lesssim\|f\|_{L_{1}\left(\mathbb{R}^{n}\right)}
$$

when $p=1$.

In order to prove Theorem 1.1, we need the following.

Theorem 3.1 Suppose $V \in R H_{q_{1}}$ with $q_{1}>n / 2, b \in \Lambda_{v}^{\theta}(\rho), \theta>0,0<v<1$. Let $0<\beta+v<n$ and let $1 \leq p<q<\infty$ satisfy $1 / q=1 / p-(\beta+v) / n$ then the inequality

$$
\begin{aligned}
\left\|\left[b, \mathcal{I}_{\beta}^{L}\right] f\right\|_{L_{q}\left(B\left(x_{0}, r\right)\right)} & \lesssim\left\|I_{\beta+v}(|f|)\right\|_{L_{q}\left(B\left(x_{0}, r\right)\right)} \\
& \lesssim r^{\frac{n}{q}} \int_{2 r}^{\infty} \frac{\|f\|_{L_{p}\left(B\left(x_{0}, t\right)\right)}}{t^{\frac{n}{q}}} \frac{d t}{t}
\end{aligned}
$$

holds for any $f \in L_{\text {loc }}^{p}\left(\mathbb{R}^{n}\right)$. Moreover, for $p=1$ the inequality

$$
\begin{aligned}
\left\|\left[b, \mathcal{I}_{\beta}^{L} f\right]\right\|_{W L_{\frac{n}{n-\beta-v}}\left(B\left(x_{0}, r\right)\right)} & \lesssim\left\|I_{\beta+v}(|f|)\right\|_{W L_{\frac{n}{n-\beta-v}}\left(B\left(x_{0}, r\right)\right)} \\
& \lesssim r^{n-\beta} \int_{2 r}^{\infty} \frac{\|f\|_{L_{1}\left(B\left(x_{0}, t\right)\right)}}{t^{n-\beta-\nu}} \frac{d t}{t}
\end{aligned}
$$

holds for any $f \in L_{\text {loc }}^{1}\left(\mathbb{R}^{n}\right)$.

Proof For arbitrary $x_{0} \in \mathbb{R}^{n}$, set $B=B\left(x_{0}, r\right)$ and $\lambda B=B\left(x_{0}, \lambda r\right)$ for any $\lambda>0$. We write $f$ as $f=f_{1}+f_{2}$, where $f_{1}(y)=f(y) \chi_{B\left(x_{0}, 2 r\right)}(y)$, and $\chi_{B\left(x_{0}, 2 r\right)}$ denotes the characteristic function of $B\left(x_{0}, 2 r\right)$. Then

$$
\begin{aligned}
\left\|\left[b, \mathcal{I}_{\beta}^{L}\right] f\right\|_{L_{q}\left(B\left(x_{0}, r\right)\right)} & \lesssim\left\|I_{\beta+v}(|f|)\right\|_{L_{q}\left(B\left(x_{0}, r\right)\right)} \\
& \leq\left\|I_{\beta+\nu} f_{1}\right\|_{L_{q}\left(B\left(x_{0}, r\right)\right)}+\left\|I_{\beta+v} f_{2}\right\|_{L_{q}\left(B\left(x_{0}, r\right)\right)} .
\end{aligned}
$$


Since $f_{1} \in L_{p}\left(\mathbb{R}^{n}\right)$ and from the boundedness of $I_{\beta+\nu}$ from $L_{p}\left(\mathbb{R}^{n}\right)$ to $L_{q}\left(\mathbb{R}^{n}\right)$ (see [31]) it follows that

$$
\begin{aligned}
\left\|I_{\beta+\nu} f_{1}\right\|_{L_{q}\left(B\left(x_{0}, r\right)\right)} & \lesssim\|f\|_{L_{p}\left(B\left(x_{0}, 2 r\right)\right)} \\
& \lesssim r^{\frac{n}{q}}\|f\|_{L_{p}\left(B\left(x_{0}, 2 r\right)\right)} \int_{2 r}^{\infty} \frac{d t}{t^{\frac{n}{q}+1}} \lesssim r^{\frac{n}{q}} \int_{2 r}^{\infty} \frac{\|f\|_{L_{p}\left(B\left(x_{0}, t\right)\right)}}{t^{\frac{n}{q}}} \frac{d t}{t} .
\end{aligned}
$$

To estimate $\left\|I_{\beta+v} f_{2}\right\|_{L_{p}\left(B\left(x_{0}, r\right)\right)}$, the obverse of $x \in B, y \in(2 B)^{c}$ implies $|x-y| \approx\left|x_{0}-y\right|$. Then by (2.2) we have

$$
\sup _{x \in B}\left|I_{\beta+\nu} f_{2}(x)\right| \lesssim \int_{(2 B)^{c}} \frac{|f(y)|}{\left|x_{0}-y\right|^{n-\beta-\nu}} d y \lesssim \sum_{k=1}^{\infty}\left(2^{k+1} r\right)^{-n+\beta} \int_{2^{k+1} B}|f(y)| d y .
$$

By Hölder's inequality we get

$$
\begin{aligned}
\sup _{x \in B}\left|I_{\beta+v} f_{2}(x)\right| & \lesssim \sum_{k=1}^{\infty}\|f\|_{L_{p}\left(2^{k+1} B\right)}\left(2^{k+1} r\right)^{-1-\frac{n}{p}+\beta} \int_{2^{k_{r}}}^{2^{k+1} r} d t \\
& \lesssim \sum_{k=1}^{\infty} \int_{2^{k_{r}}}^{2^{k+1} r} \frac{\|f\|_{L_{p}\left(B\left(x_{0}, t\right)\right)}}{t^{\frac{n}{q}}} \frac{d t}{t} \lesssim \int_{2 r}^{\infty} \frac{\|f\|_{L_{p}\left(B\left(x_{0}, t\right)\right)}}{t^{\frac{n}{q}}} \frac{d t}{t}
\end{aligned}
$$

Then

$$
\left\|I_{\beta+\nu} f_{2}\right\|_{L_{q}\left(B\left(x_{0}, r\right)\right)} \lesssim r^{\frac{n}{q}} \int_{2 r}^{\infty} \frac{\|f\|_{L_{p}\left(B\left(x_{0}, t\right)\right)}}{t^{\frac{n}{q}}} \frac{d t}{t}
$$

holds for $1 \leq p<n / \beta$. Therefore, by (3.1) and (3.3) we get

$$
\left\|I_{\beta+v}(|f|)\right\|_{L_{q}\left(B\left(x_{0}, r\right)\right)} \lesssim r^{\frac{n}{q}} \int_{2 r}^{\infty} \frac{\|f\|_{L_{p}\left(B\left(x_{0}, t\right)\right)}}{t^{\frac{n}{q}}} \frac{d t}{t}
$$

for $1<p<n / \beta$.

When $p=1$, by the boundedness of $I_{\beta+\nu}$ from $L_{1}\left(\mathbb{R}^{n}\right)$ to $W L_{\frac{n}{n-\beta-\nu}}\left(\mathbb{R}^{n}\right)$, we get

$$
\left\|I_{\beta+\nu} f_{1}\right\|_{W L_{\frac{n}{n-\beta-v}}\left(B\left(x_{0}, r\right)\right)} \lesssim\|f\|_{L_{1}\left(B\left(x_{0}, 2 r\right)\right)} \lesssim r^{n-\beta-\nu} \int_{2 r}^{\infty} \frac{\|f\|_{L_{1}\left(B\left(x_{0}, t\right)\right)}}{t^{n-\beta-\nu}} \frac{d t}{t} .
$$

By (3.3) we have

$$
\begin{aligned}
\left\|I_{\beta+\nu} f_{2}\right\|_{W L} \frac{n}{n-\beta-v}\left(B\left(x_{0}, r\right)\right) & \leq\left\|I_{\beta+\nu} f_{2}\right\|_{L_{\frac{n}{n-\beta-v}}\left(B\left(x_{0}, 2 r\right)\right)} \\
& \lesssim r^{n-\beta-\nu} \int_{2 r}^{\infty} \frac{\|f\|_{L_{1}\left(B\left(x_{0}, t\right)\right)}}{t^{n-\beta-\nu}} \frac{d t}{t} .
\end{aligned}
$$

Then

$$
\left\|I_{\beta+\nu}(|f|)\right\|_{W L \frac{n}{n-\beta-v}}\left(B\left(x_{0}, r\right)\right) \lesssim r^{n-\beta-\nu} \int_{2 r}^{\infty} \frac{\|f\|_{L_{1}\left(B\left(x_{0}, t\right)\right)}}{t^{n-\beta-v}} \frac{d t}{t} .
$$


Proof of Theorem 1.1 From Lemma 2.6, we have

$$
\frac{1}{\operatorname{ess} \inf _{t<s<\infty} \varphi_{1}(x, s) s^{\frac{n}{p}}}=\underset{t<s<\infty}{\operatorname{ess} \sup } \frac{1}{\varphi_{1}(x, s) s^{\frac{n}{p}}} .
$$

Note the fact that $\|f\|_{L_{p}\left(B\left(x_{0}, t\right)\right)}$ is a nondecreasing function of $t$, and $f \in M_{p, \varphi_{1}}^{\alpha, V}$, then

$$
\begin{aligned}
\frac{\left(1+\frac{t}{\rho\left(x_{0}\right)}\right)^{\alpha}\|f\|_{L_{p}\left(B\left(x_{0}, t\right)\right)}}{\operatorname{essinf}_{t<s<\infty} \varphi_{1}(x, s) s^{\frac{n}{p}}} & \lesssim \operatorname{ess~sup}_{t<s<\infty} \frac{\left(1+\frac{t}{\rho\left(x_{0}\right)}\right)^{\alpha}\|f\|_{L_{p}\left(B\left(x_{0}, t\right)\right)}}{\varphi_{1}(x, s) s^{\frac{n}{p}}} \\
& \lesssim \sup _{0<s<\infty} \frac{\left(1+\frac{s}{\rho\left(x_{0}\right)}\right)^{\alpha}\|f\|_{L_{p}\left(B\left(x_{0}, s\right)\right)}}{\varphi_{1}(x, s) s^{\frac{n}{p}}} \lesssim\|f\|_{M_{p, \varphi_{1}}^{\alpha}, V}
\end{aligned}
$$

Since $\alpha \geq 0$, and $\left(\varphi_{1}, \varphi_{2}\right)$ satisfies the condition (1.5),

$$
\begin{aligned}
\int_{2 r}^{\infty} & \frac{\|f\|_{L_{p}\left(B\left(x_{0}, t\right)\right)}}{t^{\frac{n}{q}}} \frac{d t}{t} \\
\quad & \int_{2 r}^{\infty} \frac{\left(1+\frac{t}{\rho\left(x_{0}\right)}\right)^{\alpha}\|f\|_{L_{p}\left(B\left(x_{0}, t\right)\right)}}{\operatorname{essinf}_{t<s<\infty} \varphi_{1}(x, s) s^{\frac{n}{p}}} \frac{\operatorname{essinf}_{t<s<\infty} \varphi_{1}(x, s) s^{\frac{n}{p}}}{\left(1+\frac{t}{\rho\left(x_{0}\right)}\right)^{\alpha} t^{\frac{n}{q}}} \frac{d t}{t} \\
& \lesssim\|f\|_{M_{p, \varphi_{1}}^{\alpha, V}} \int_{2 r}^{\infty} \frac{\operatorname{essinf}_{t<s<\infty} \varphi_{1}(x, s) s^{\frac{n}{p}}}{\left(1+\frac{t}{\rho\left(x_{0}\right)}\right)^{\alpha} t^{\frac{n}{q}}} \frac{d t}{t} \\
& \lesssim\|f\|_{M_{p, \varphi_{1}}^{\alpha, V}}\left(1+\frac{r}{\rho\left(x_{0}\right)}\right)^{-\alpha} \int_{r}^{\infty} \frac{\operatorname{essinf}_{t<s<\infty} \varphi_{1}(x, s) s^{\frac{n}{p}}}{t^{\frac{n}{q}}} \frac{d t}{t} \\
& \lesssim\|f\|_{M_{p, \varphi_{1}}^{\alpha, V}}\left(1+\frac{r}{\rho\left(x_{0}\right)}\right)^{-\alpha} \varphi_{2}\left(x_{0}, r\right) .
\end{aligned}
$$

Then by Theorem 3.1 we get

$$
\begin{aligned}
\left\|\left[b, \mathcal{I}_{\beta}^{L}\right] f\right\|_{M_{q, \varphi_{2}}^{\alpha, V}} & \lesssim\left\|I_{\beta+\nu}(|f|)\right\|_{M_{q, \varphi_{2}}^{\alpha, V}} \\
& \lesssim \sup _{x_{0} \in \mathbb{R}^{n}, r>0}\left(1+\frac{r}{\rho\left(x_{0}\right)}\right)^{\alpha} \varphi_{2}\left(x_{0}, r\right)^{-1} r^{-n / q}\left\|I_{\beta+v}(|f|)\right\|_{L_{p}\left(B\left(x_{0}, r\right)\right)} \\
& \lesssim \sup _{x_{0} \in \mathbb{R}^{n}, r>0}\left(1+\frac{r}{\rho\left(x_{0}\right)}\right)^{\alpha} \varphi_{2}\left(x_{0}, r\right)^{-1} \int_{2 r}^{\infty} \frac{\|f\|_{L_{p}\left(B\left(x_{0}, t\right)\right)}}{t^{\frac{n}{q}}} \frac{d t}{t} \\
& \lesssim\|f\|_{M_{p, \varphi_{1}}^{\alpha, V}} .
\end{aligned}
$$

Let $q=\frac{n}{n-\beta-v}$, similar to the estimates of (3.5) we have

$$
\int_{2 r}^{\infty} \frac{\|f\|_{L_{1}\left(B\left(x_{0}, t\right)\right)}}{t^{n-\beta-v}} \frac{d t}{t} \lesssim\|f\|_{M_{1, \varphi_{1}}^{\alpha, V}}\left(1+\frac{r}{\rho\left(x_{0}\right)}\right)^{-\alpha} \varphi_{2}\left(x_{0}, r\right) .
$$

Thus by Theorem 3.1 we get

$$
\begin{aligned}
\left\|\left[b, \mathcal{I}_{\beta}^{L}\right] f\right\|_{W M^{\alpha, V} \frac{n}{n-\beta-v}, \varphi_{2}} & \lesssim\left\|I_{\beta+\nu}(|f|)\right\|_{W M_{\frac{n}{n-\beta-v}, \varphi_{2}}^{\alpha, V}} \\
& \lesssim \sup _{x_{0} \in \mathbb{R}^{n}, r>0}\left(1+\frac{r}{\rho\left(x_{0}\right)}\right)^{\alpha} \varphi_{2}\left(x_{0}, r\right)^{-1} r^{\beta-n}\left\|I_{\beta+\nu}(|f|)\right\|_{W L_{\frac{n}{n-\beta-v}}\left(B\left(x_{0}, r\right)\right)}
\end{aligned}
$$




$$
\begin{aligned}
& \lesssim \sup _{x_{0} \in \mathbb{R}^{n}, r>0}\left(1+\frac{r}{\rho\left(x_{0}\right)}\right)^{\alpha} \varphi_{2}\left(x_{0}, r\right)^{-1} \int_{2 r}^{\infty} \frac{\|f\|_{L_{1}\left(B\left(x_{0}, t\right)\right)}}{t^{n-\beta-v}} \frac{d t}{t} \\
& \lesssim\|f\|_{M_{1, \varphi_{1}}^{\alpha, V}}
\end{aligned}
$$

\section{Proof of Theorem 1.2}

The statement is derived from the estimate (3.4). The estimation of the norm of the operator, that is, the boundedness in the non-vanishing space, immediately follows by Theorem 1.1. So we only have to prove that

$$
\lim _{r \rightarrow 0} \sup _{x \in \mathbb{R}^{n}} \mathfrak{A}_{p, \varphi_{1}}^{\alpha, V}(f ; x, r)=0 \Rightarrow \lim _{r \rightarrow 0} \sup _{x \in \mathbb{R}^{n}} \mathfrak{A}_{q, \varphi_{2}}^{\alpha, V}\left(\left[b, \mathcal{I}_{\beta}^{L}\right] f ; x, r\right)=0
$$

and

$$
\lim _{r \rightarrow 0} \sup _{x \in \mathbb{R}^{n}} \mathfrak{A}_{1, \varphi_{1}}^{\alpha, V}(f ; x, r)=0 \Rightarrow \lim _{r \rightarrow 0} \sup _{x \in \mathbb{R}^{n}} \mathfrak{A}_{n /(n-\beta), \varphi_{2}}^{W, \alpha, V}\left(\left[b, \mathcal{I}_{\beta}^{L}\right] f ; x, r\right)=0
$$

To show that $\sup _{x \in \mathbb{R}^{n}}\left(1+\frac{r}{\rho(x)}\right)^{\alpha} \varphi_{2}(x, r)^{-1} r^{-n / p}\left\|\left[b, \mathcal{I}_{\beta}^{L}\right] f\right\|_{L_{q}(B(x, r))}<\varepsilon$ for small $r$, we split the right-hand side of (3.4):

$$
\left(1+\frac{r}{\rho(x)}\right)^{\alpha} \varphi_{2}(x, r)^{-1} r^{-n / p}\left\|\left[b, \mathcal{I}_{\beta}^{L}\right] f\right\|_{L_{q}(B(x, r))} \leq C\left[I_{\delta_{0}}(x, r)+J_{\delta_{0}}(x, r)\right]
$$

where $\delta_{0}>0$ (we may take $\delta_{0}>1$ ), and

$$
I_{\delta_{0}}(x, r):=\frac{\left(1+\frac{r}{\rho(x)}\right)^{\alpha}}{\varphi_{2}(x, r)} \int_{r}^{\delta_{0}} t^{-\frac{n}{q}-1}\|f\|_{L_{p}(B(x, t))} d t
$$

and

$$
J_{\delta_{0}}(x, r):=\frac{\left(1+\frac{r}{\rho(x)}\right)^{\alpha}}{\varphi_{2}(x, r)} \int_{\delta_{0}}^{\infty} t^{-\frac{n}{q}-1}\|f\|_{L_{p}(B(x, t))} d t
$$

and it is supposed that $r<\delta_{0}$. We use the fact that $f \in V M_{p, \varphi_{1}}^{\alpha, V}\left(\mathbb{R}^{n}\right)$ and choose any fixed $\delta_{0}>0$ such that

$$
\sup _{x \in \mathbb{R}^{n}}\left(1+\frac{t}{\rho(x)}\right)^{\alpha} \varphi_{1}(x, t)^{-1} t^{-n / p}\|f\|_{L_{p}(B(x, t))}<\frac{\varepsilon}{2 C C_{0}}
$$

where $C$ and $C_{0}$ are constants from (1.6) and (4.3). This allows one to estimate the first term uniformly in $r \in\left(0, \delta_{0}\right)$ :

$$
\sup _{x \in \mathbb{R}^{n}} C I_{\delta_{0}}(x, r)<\frac{\varepsilon}{2}, \quad 0<r<\delta_{0}
$$

The estimation of the second term now my be made already by the choice of $r$ sufficiently small. Indeed, thanks to the condition (2.6) we have

$$
J_{\delta_{0}}(x, r) \leq c_{\sigma_{0}} \frac{\left(1+\frac{r}{\rho(x)}\right)^{\alpha}}{\varphi_{1}(x, r)}\|f\|_{V M_{,, \varphi_{1}}^{\alpha, V}}
$$


where $c_{\sigma_{0}}$ is the constant from (1.3). Then by (2.6) it suffices to choose $r$ small enough such that

$$
\sup _{x \in \mathbb{R}^{n}} \frac{\left(1+\frac{r}{\rho(x)}\right)^{\alpha}}{\varphi_{2}(x, r)} \leq \frac{\varepsilon}{2 c_{\sigma_{0}}\|f\|_{V M_{p, \varphi_{1}}^{\alpha, V}}},
$$

which completes the proof of (4.1).

The proof of (4.2) is similar to the proof of (4.1).

\section{Conclusions}

In this paper, we study the boundedness of the commutators $\left[b, \mathcal{I}_{\beta}^{L}\right]$ with $b \in \Lambda_{v}^{\theta}(\rho)$ on local generalized Morrey spaces $L M_{p, \varphi}^{\alpha, V,\left\{x_{0}\right\}}$, generalized Morrey spaces $M_{p, \varphi}^{\alpha, V}$ and vanishing generalized Morrey spaces $V M_{p, \varphi}^{\alpha, V}$ associated with the Schrödinger operator, respectively. When $b$ belongs to $\Lambda_{v}^{\theta}(\rho)$ with $\theta>0,0<v<1$ and $\left(\varphi_{1}, \varphi_{2}\right)$ satisfies some conditions, we show that the commutator operator $\left[b, \mathcal{I}_{\beta}^{L}\right]$ are bounded from $L M_{p, \varphi_{1}}^{\alpha, V,\left\{x_{0}\right\}}$ to $L M_{q, \varphi_{2}}^{\alpha, V,\left\{x_{0}\right\}}$, from $M_{p, \varphi_{1}}^{\alpha, V}$ to $M_{q, \varphi_{2}}^{\alpha, V}$ and from $V M_{p, \varphi_{1}}^{\alpha, V}$ to $V M_{q, \varphi_{2}}^{\alpha, V}, 1 / p-1 / q=(\beta+v) / n$.

Our results about the boundedness of $\left[b, \mathcal{I}_{\beta}^{L}\right]$ with $b \in \Lambda_{v}^{\theta}(\rho)$ from $L M_{p, \varphi_{1}}^{\alpha, V,\left\{x_{0}\right\}}$ to $L M_{q, \varphi_{2}}^{\alpha, V,\left\{x_{0}\right\}}$ (Theorem 1.1) are based on the local estimate for the commutators $\left[b, \mathcal{I}_{\beta}^{L}\right]$ (Theorem 3.1).

\section{Acknowledgements}

The authors thank the referees for careful reading the paper and useful comments.

\section{Funding}

The research of A. Akbulut was partially supported by the grant of Ahi Evran University Scientific Research Project (FEF.A4.17.020). The research of V.S. Guliyev was partially supported by the grant of Ahi Evran University Scientific Research Project (FEF.A4.17.008), by the grant of 1st Azerbaijan-Russia Joint Grant Competition (the Agreement number No. 18-51-06005) and by the Ministry of Education and Science of the Russian Federation (Agreement number: 02.a03.21.0008).

\section{Availability of data and materials}

Not applicable.

\section{Competing interests}

The authors declare that they have no competing interests.

\section{Authors' contributions}

This work was carried out in collaboration between all authors. VSG raised these interesting problems in the research. VSG and AA proved the theorems, interpreted the results and wrote the article. All authors defined the research theme, read and approved the manuscript.

\section{Author details}

${ }^{1}$ Department of Mathematics, Ahi Evran University, Kirsehir, Turkey. ${ }^{2}$ S.M. Nikolskii Institute of Mathematics at RUDN University, Moscow, Russia. ${ }^{3}$ Institute of Mathematics and Mechanics of NAS of Azerbaijan, Baku, Azerbaijan.

\section{Publisher's Note}

Springer Nature remains neutral with regard to jurisdictional claims in published maps and institutional affiliations.

Received: 22 March 2018 Accepted: 17 May 2018 Published online: 22 May 2018

References

1. Akbulut, A., Eroglu, A., Najafov, A.M.: Some embedding theorems on the Nikolskii-Morrey type spaces. Adv. Anal. 1(1), $18-26(2016)$

2. Akbulut, A., Guliyev, V.S., Omarova, M.N.: Marcinkiewicz integrals associated with Schrödinger operators and their commutators on vanishing generalized Morrey spaces. Bound. Value Probl. 2017, 121 (2017)

3. Akbulut, A., Kuzu, O.: Marcinkiewicz integrals associated with Schrödinger operator on generalized Morrey spaces. J. Math. Inequal. 8(4), 791-801 (2014)

4. Alvarez, J., Lakey, J., Guzman-Partida, M.: Spaces of bounded $\lambda$-central mean oscillation, Morrey spaces, and $\lambda$-central Carleson measures. Collect. Math. 51(1), 1-47 (2000) 
5. Bongioanni, B., Harboure, E., Salinas, O.: Commutators of Riesz transforms related to Schödinger operators. J. Fourier Anal. Appl. 17(1), 115-134 (2011)

6. Bui, T.: Weighted estimates for commutators of some singular integrals related to Schrödinger operator. Bull. Sci. Math. 138(2), 270-292 (2014)

7. Cao, X., Chen, D.: The boundedness of Toeplitz-type operators on vanishing-Morrey spaces. Anal. Theory Appl. 27(4), 309-319 (2011)

8. Chanillo, S.: A note on commutators. Indiana Univ. Math. J. 31(1), 7-16 (1982)

9. Chiarenza, F., Frasca, M.: Morrey spaces and Hardy-Littlewood maximal function. Rend. Mat. 7, 273-279 (1987)

10. Di Fazio, G., Ragusa, M.A.: Interior estimates in Morrey spaces for strong solutions to nondivergence form equations with discontinuous coefficients. J. Funct. Anal. 112, 241-256 (1993)

11. Fan, D., Lu, S., Yang, D.: Boundedness of operators in Morrey spaces on homogeneous spaces and its applications. Acta Math. Sin. New Ser. 14, 625-634 (1998)

12. Giannetti, F., Passarelli di Napoli, A., Ragusa, M.A., Tachikawa, A.: Partial regularity for minimizers of a class of non autonomous functionals with nonstandard growth. Calc. Var. Partial Differ. Equ. 56(6), Article ID 153 (2017)

13. Guliyev, V.S.: Integral operators on function spaces on the homogeneous groups and on domains in $\mathbb{R}^{n}$. Doctor's degree dissertation. Mat. Inst. Steklov, Moscow (1994) 329 pp. (in Russian)

14. Guliyev, V.S.: Function spaces, integral operators and two weighted inequalities on homogeneous groups. Some applications. Casioglu, Baku (1999) 332 pp. (in Russian)

15. Guliyev, V.S.: Boundedness of the maximal, potential and singular operators in the generalized Morrey spaces. J. Inequal. Appl. 2009, Article ID 503948 (2009)

16. Guliyev, V.S.: Local generalized Morrey spaces and singular integrals with rough kernel. Azerb. J. Math. 3(2), 79-94 (2013)

17. Guliyev, V.S.: Generalized local Morrey spaces and fractional integral operators with rough kernel. J. Math. Sci. (N.Y.) 193(2), 211-227 (2013)

18. Guliyev, V.S.: Function spaces and integral operators associated with Schrödinger operators: an overview. Proc. Inst. Math. Mech. Natl. Acad. Sci. Azerb. 40, 178-202 (2014)

19. Guliyev, V.S., Aliyev, S.S., Karaman, T., Shukurov, P.: Boundedness of sublinear operators and commutators on generalized Morrey spaces. Integral Equ. Oper. Theory 71(3), 327-355 (2011)

20. Guliyev, V.S., Guliyev, R.V., Omarova, M.N.: Riesz transforms associated with Schrödinger operator on vanishing generalized Morrey spaces. Appl. Comput. Math. 17(1), 56-71 (2018)

21. Guliyev, V.S., Omarova, M.N., Ragusa, M.A., Scapellato, A.: Commutators and generalized local Morrey spaces. J. Math Anal. Appl. 457(2), 1388-1402 (2018)

22. Liu, Y., Sheng, J.: Some estimates for commutators of Riesz transforms associated with Schrödinger operators. J. Math Anal. Appl. 419, 298-328 (2014)

23. Mizuhara, T.: Boundedness of some classical operators on generalized Morrey spaces. In: Igari, S. (ed.) Harmonic Analysis. ICM 90 Satellite Proceedings, pp. 183-189. Springer, Tokyo (1991)

24. Morrey, C.: On the solutions of quasi-linear elliptic partial differential equations. Trans. Am. Math. Soc. 43, 126-166 (1938)

25. Nakai, E.: Hardy-Littlewood maximal operator, singular integral operators and the Riesz potentials on generalized Morrey spaces. Math. Nachr. 166, 95-103 (1994)

26. Paluszyński, M.: Characterization of the Besov spaces via the commutator operator of Coifman, Rochberg and Weiss. Indiana Univ. Math. J. 44(1), 1-17 (1995)

27. Ragusa, M.A.: Commutators of fractional integral operators on vanishing-Morrey spaces. J. Glob. Optim. 40(1-3), 361-368 (2008)

28. Samko, N.: Maximal, potential and singular operators in vanishing generalized Morrey spaces. J. Glob. Optim. 57(4), 1385-1399 (2013)

29. Shen, Z.: $L_{p}$ estimates for Schrödinger operators with certain potentials. Ann. Inst. Fourier (Grenoble) 45(2), 513-546 (1995)

30. Softova, L.: Singular integrals and commutators in generalized Morrey spaces. Acta Math. Sin. Engl. Ser. 22(3), 757-766 (2006)

31. Stein, E.M.: Harmonic Analysis: Real-Variable Methods, Orthogonality, and Oscillatory Integrals. Princeton Univ. Press, Princeton (1993)

32. Tang, L., Dong, J.: Boundedness for some Schrödinger type operator on Morrey spaces related to certain nonnegative potentials. J. Math. Anal. Appl. 355, 101-109 (2009)

33. Vitanza, C.: Functions with vanishing Morrey norm and elliptic partial differential equations. In: Proceedings of Methods of Real Analysis and Partial Differential Equations, Capri, pp. 147-150. Springer, Berlin (1990)

34. Wheeden, R., Zygmund, A.: Measure and Integral, an Introduction to Real Analysis. Pure and Applied Mathematics, vol. 43. Dekker, New York (1977)

35. Xiao, J.: A new perspective on the Riesz potential. Adv. Nonlinear Anal. 6(3), 317-326 (2017) 